\begin{tabular}{l|l|l|l|l} 
Revue suisse Zool. & Tome 90 & Fasc. 4 & p. 929-934 & Genève, décembre 1983
\end{tabular}

\title{
Deux nouvelles espèces du genre Stachorutes Dallai, 1973 (Collembola)
}

\author{
par \\ Louis DEHARVENG * et Charles LIENHARD **
}

Avec 15 figures

Abstract

Two new species of the genus Stachorutes Dallai, 1973 (Collembola). - The two new species described in the mentioned genus have been collected at high altitude in the Oriental Pyrenees ( $S$. longirostris n. sp.) and in the Swiss Alps (S. scherae n. sp.).

\section{Le genre Stachorutes Dallai, 1973}

Diagnose: Moins de $8+8$ cornéules $(2+2$ à $5+5)$.

Furca réduite.

Capitulum maxillaire styliforme, mandibule grêle à 2 ou 3 dents.

Organe postantennaire présent, à tubercules sur un seul rang.

Labium sans soie $\mathrm{L}$ distale.

Ant. IV avec 6 sensilles S épaissies subégales (S1, S2, S3, S4, S7 et S8) et une microsensille dorso-latérale $\mathrm{m}$.

Pas de râpe sensorielle sur ant. IV.

Ant. III et IV soudés sur leur face dorsale.

Chétotaxie dorsale réduite: soie a 0 absente sur la tête; d1 représentée par une soie impaire; $\mathrm{m} 1$ absente sur th.II, th.III et abd.IV.

* Laboratoire d'écologie et écophysiologie des insectes, LA 333 du C.N.R.S., 118, route de Narbonne, F-31062 Toulouse Cedex.

** Muséum d'Histoire naturelle, Case postale 434, CH-1211 Genève 6. (Le travail sur le terrain de cet auteur a été soutenu par le Fonds national suisse pour la recherche scientifique, requête $n^{0} 3.628-0.75$ ). 
Discussion : Le genre Stachorutes présente, comme le genre Furculanurida Massoud 1967, un nombre de cornéules réduit; la régression de l'appareil furcal, observée chez tous les Stachorutes, n'existe pour Furculanurida que chez l'espèce perplexa (Salmon, 1956). Ces similitudes, concernant des organes extrêmement sensibles aux évolutions régressives, ne nous semblent pas refléter une réelle affinité des deux genres, qui diffèrent par d'importants caractères au niveau de la chétotaxie antennaire, en particulier l'organite d'ant. III (cf. MASSOUD 1967, p. 232).

Les données chétotaxiques concernant les espèces du genre Furculanurida sont malheureusement peu nombreuses. En ce qui concerne le genre Stachorutes, la morphologie et la forme des soies, leur disposition sur la tête, les tergites, les sternites, les antennes et le labium ainsi que la forme de la maxille et de la mandibule sont identiques à ce qu'on peut observer chez Pseudachorutes parvulus Börner, 1901.

Dans l'état actuel de nos connaissances, le genre Stachorutes peut donc être considéré comme une lignée issue, par évolution régressive des yeux et de la furca, de formes voisines de Pseudachorutes parvulus.

Liste des espèces:

S. dematteisi Dallai, 1973 (Italie), espèce type.

S. longirostris, n. sp. (Pyrénées).

S. scherae, n. sp. (Alpes).

Micranurida ashrafi Yosii, 1966 du Népal devra peut-être être rattachée au genre Stachorutes, mais nous ne possédons aucune précision sur sa chétotaxie dorsale et antennaire.

\section{Stachorutes longirostris $\mathrm{n}$. $\mathrm{sp}$.}

Matériel: + holotype, 1 ô et 1 q paratypes. France: Pyrénées Orientales, Canigou: Cirque des Cortalets, $2500 \mathrm{~m}$, humus près d'un névé, 8-VI-1973. Holotype au Muséum d'Histoire naturelle de Genève. Paratypes dans la collection DeHARveng (Toulouse).

Description: 0,65 à $0,75 \mathrm{~mm}$. Grisâtre à bleuâtre. Habitus de Pseudachorutes parvulus. Soies dorsales courtes, subégales, les sensilles un peu plus longues que les soies ordinaires. Grain tégumentaire secondaire hémisphérique, légèrement plus grand que les embases de soies.

Pièces buccales réduites, la mandibule grêle à 2 ou 3 dents, le capitulum maxillaire styliforme. Labium sans soie L distale; labre ogival, pointu, bien sclérifié dans sa partie ventro-apicale (fig. 3). Cône buccal très long. Plage oculaire avec 5 cornéules subégales; organe postantennaire ovalaire, comportant 9-10 tubercules simples, subégaux sur un seul rang (fig. 2). Antennes assez trapues. Ant. I porte 7 soies ordinaires, ant. II en porte 12. Ant. III possède 17-18 soies ordinaires et un organite classique, composé de 2 microsensilles «si » globuleuses, 2 longues sensilles de garde subégales sgd et sgv. et une microsensille accessoire "sa » ventrolatérale. Ant. IV porte dorsalement des soies mousses dressées, moyennes, quelques soies ordinaires (i centrale et quelquesunes distales), 6 sensilles épaissies $\mathrm{S} 1$ à $\mathrm{S} 4, \mathrm{~S} 7$ et $\mathrm{S} 8$, une microsensille dorso-externe $\mathrm{m}$, un organite distal " or » très petit, enfin une vésicule apicale entière ou faiblement trilobée (fig. 1). Chez un exemplaire, la vésicule apicale est entière sur une antenne et trilobée sur l'autre.

Chétotaxie dorsale réduite avec des assymétries fréquentes (fig. 4). Les sensilles $S$ se trouvent en position $3,3 / 4,4,4,4,2$ de th. II à abd. V. La formule des groupes de soies dorso-internes est $1,3,3 / 3,3,3,3$ de th. I à abd. IV. Aucun macrochète n'est différencié, même sur abd. VI. La chétotaxie ventrale de l'abdomen est représentée sur 
a fig. 5 . Le tube ventral porte $4+4$ soies, abd.II $4+4$ soies, abd. III $8+8$ soies. La présence de $1+1$ soies sur chaque lèvre de la plaque génitale femelle constitue un caractère remarquable. Abd. V porte une rangée de 4-5 + 4-5 soies ventrales. Le ster-

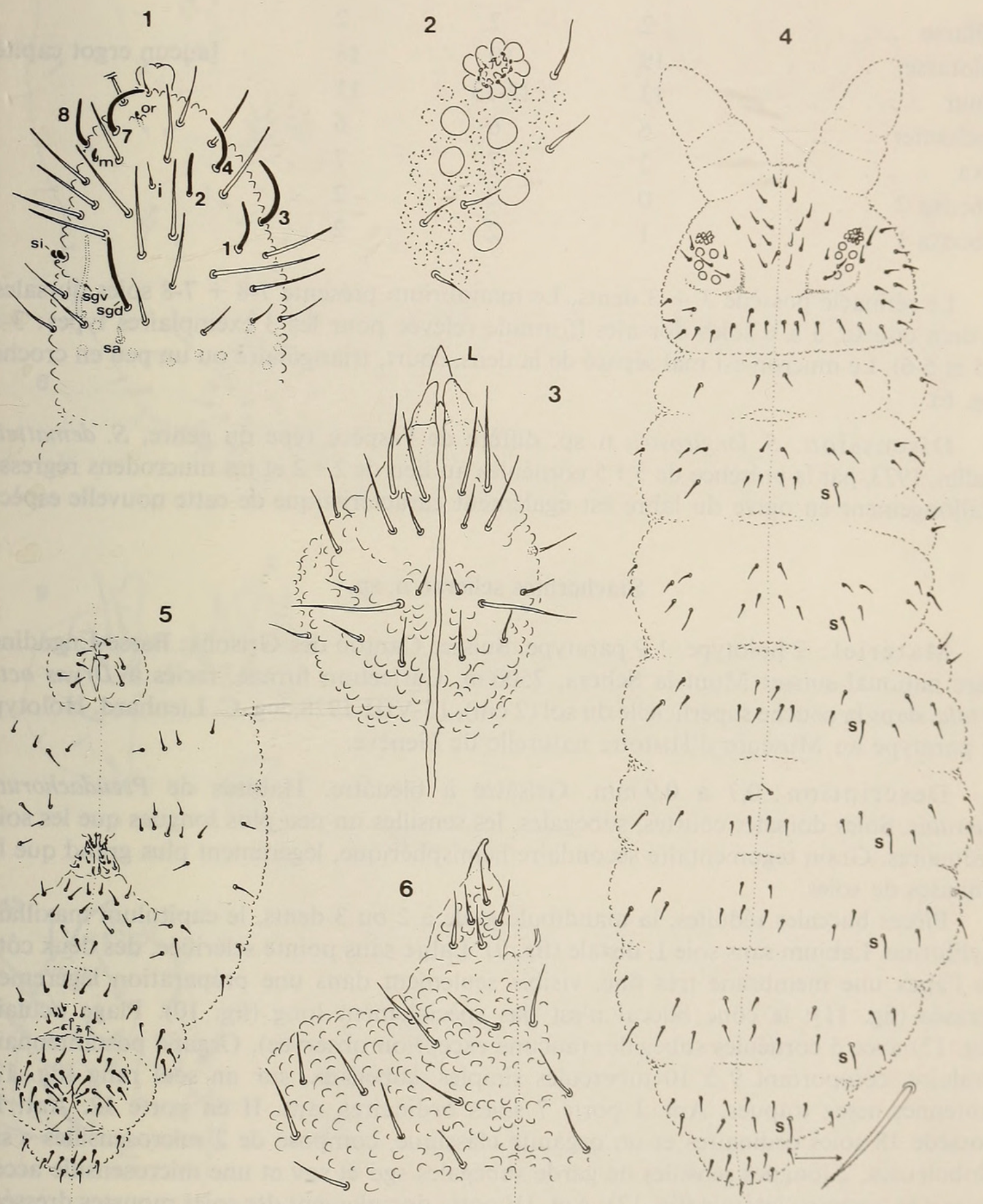

FIG. 1-6.

Stachorutes longirostris n. sp.: 1. Articles III et IV de l'antenne en vue dorsale. Les sensilles S et les embases des soies ordinaires d'ant. III sont indiquées en pointillés. 2. Plage oculaire et organe postantennaire. 3. Labium et extrémité du labre (L). 4. Chétotaxie dorsale (S: sensilles S des tergites). 5. Chétotaxie ventrale. 6. Mucrodens et manubrium. 
nite d'abdomen VI est muni de 13-14 $+13-14$ soies et 2 microchètes sur chaque valve anale.

La formule chétotaxique des pattes est la suivante:

\begin{tabular}{|c|c|c|c|c|c|}
\hline & & PI & PII & PIII & \\
\hline prétarse. & . . . . & 2 & 2 & 2 & \\
\hline tibiotarse & . . . & 19 & 19 & 18 & [aucun ergot capité] \\
\hline fémur. . & 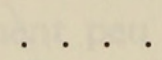 & 13 & $12-13$ & 11 & \\
\hline trochanter & . . . & 6 & 6 & 6 & \\
\hline coxa. & . . . & 3 & 7 & 7 & \\
\hline subcoxa 2 & . . . . & 0 & 2 & 2 & \\
\hline subcoxa 1 & . . . . & 1 & 2 & 2 & \\
\hline
\end{tabular}

Le rétinacle possède $3+3$ dents. Le manubrium présente 7-8 $+7-8$ soies dorsales; la dens courte, 3 à 6 soies dorsales (formule relevée pour les 3 exemplaires types: 3-5, 4-5 et 5-6). Le mucron est mal séparé de la dens, court, triangulaire ou un peu en crochet (fig. 6).

Discussion. S. longirostris n. sp. diffère de l'espèce type du genre, S. dematteisi Dallai, 1973, par la présence de $5+5$ cornéules au lieu de $2+2$ et un mucrodens régressé. L'allongement en ogive du labre est également caractéristique de cette nouvelle espèce.

\section{Stachorutes scherae n. sp.}

Matériel : + holotype, 1 q paratype. Suisse: Canton des Grisons: Basse-Engadine: Parc national suisse: Munt la Schera, $2500 \mathrm{~m}$, Caricetum firmae, faciès à Dryas octopetala, dans la couche superficielle du sol $(2 \mathrm{~cm}), 15-$ VIII-1978, leg. C. Lienhard. Holotype et paratype au Muséum d'Histoire naturelle de Genève.

Description: 0,7 à $0,9 \mathrm{~mm}$. Grisâtre à bleuâtre. Habitus de Pseudachorutes parvulus. Soies dorsales courtes, subégales, les sensilles un peu plus longues que les soies ordinaires. Grain tégumentaire secondaire hémisphérique, légèrement plus grand que les embases de soies.

Pièces buccales réduites, la mandibule grèle à 2 ou 3 dents, le capitulum maxillaire styliforme. Labium sans soie L distale (fig. 9). Labre sans pointe sclérifiée, des deux côtés de l'apex une membrane très fine, visible seulement dans une préparation légèrement écrasée (fig. 11); le cône buccal n'est pas spécialement long (fig. 10). Plage oculaire (fig. 12) avec 5 cornéules subégales (aucune exception observée). Organe postantennaire ovalaire, comportant 9 à 10 tubercules simples, subégaux, sur un seul rang (fig. 12). Antennes assez trapues. Ant. I porte 7 soies ordinaires, ant. II en porte 12. Ant. III possède 18 soies ordinaires et un organite classique, composé de 2 microsensilles «si » globuleuses, 2 longues sensilles de garde subégales sgd et sgv et une microsensille accessoire « sa » ventro-latérale (fig. 13). Ant. IV porte dorsalement des soies mousses dressées, moyennes, quelques soies ordinaires (i centrale et quelques unes distales), 6 sensilles épaissies $\mathrm{S} 1$ à $\mathrm{S} 4, \mathrm{~S} 7$ et $\mathrm{S} 8$, une microsensille dorso-externe $\mathrm{m}$, un organite distal « or » petit, enfin une vésicule apicale entière (fig. 13).

Chétotaxie dorsale réduite (fig. 7). Les sensilles $\mathrm{S}$ se trouvent en position 3,3/4,4,4,4,2 de th. II à abd. V. La formule des groupes de soies dorso-internes est 1,3,3/3,3,3,3 de th. I à abd. IV. Aucun macrochète n'est différencié, même sur abd. VI. La chétotaxie ventrale 


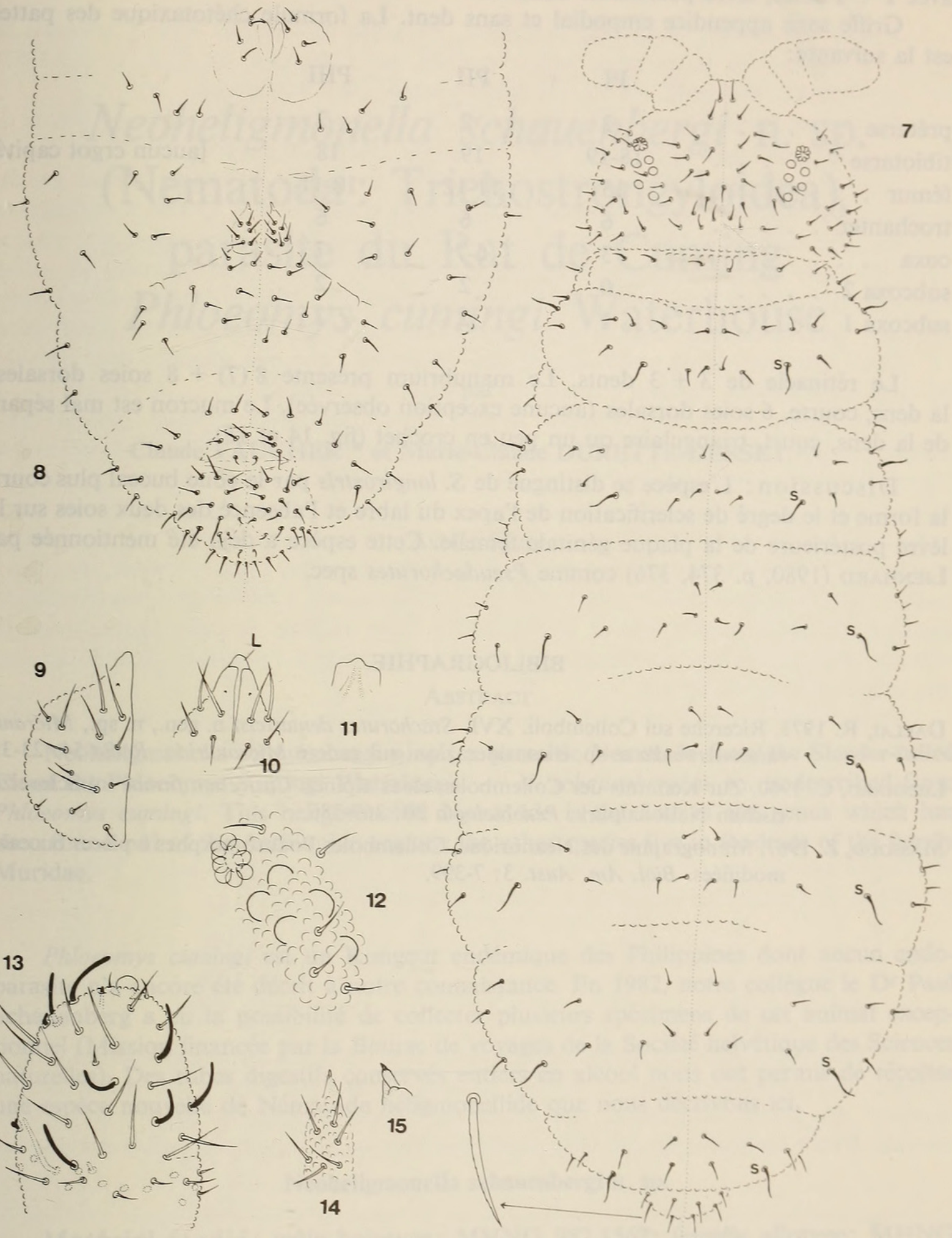

FIG. 7-15.

Stachorutes scherae n. sp.: 7. Chétotaxie dorsale (S: sensilles S des tergites). 8. Chétotaxie ventrale. 9. Labium. 10. Partie apicale du labium et extrémité du labre (L). 11. Extrémité du labre (dans préparation légèrement écrasée). 12. Plage oculaire et organe postantennaire. 13. Articles III et IV de l'antenne en vue dorsale. Les sensilles $\mathrm{S}$ et les embases des soies ordinaires d'ant. III sont indiquées en pointillés. 14. Mucrodens en vue dorsale. 15. Mucro en vue ventrale. 
de l'abdomen est représentée sur la fig. 8. Lèvre antérieure de la plaque génitale femelle avec $1+1$ soies, lèvre postérieure sans soies.

Griffe sans appendice empodial et sans dent. La formule chétotaxique des pattes est la suivante:

\begin{tabular}{|c|c|c|c|c|c|}
\hline & & PI & PII & PIII & \\
\hline prétarse. & . . . . & 2 & 2 & 2 & \\
\hline tibiotarse & . . . . & $18-19$ & 19 & 18 & [aucun ergot capité] \\
\hline fémur. . & 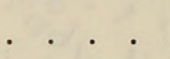 & 13 & $11-12$ & $10-11$ & \\
\hline trochanter & . . . . & 6 & 6 & 6 & . \\
\hline coxa & . . & 3 & $6-7$ & 7 & $\cdot$ \\
\hline subcoxa 2 & . . . . & 0 & 2 & 2 & \\
\hline subcoxa 1 & . . . . & 1 & 2 & 2 & \\
\hline
\end{tabular}

Le rétinacle de $3+3$ dents. Le manubrium présente $8(7)+8$ soies dorsales; la dens, courte, 6 soies dorsales (aucune exception observée). Le mucron est mal séparé de la dens, court, triangulaire ou un peu en crochet (fig. 14 et 15).

Discussion: L'espèce se distingue de $S$. longirostris par le cône buccal plus court, la forme et le degré de sclerification de l'apex du labre et l'absence des deux soies sur la lèvre postérieure de la plaque génitale femelle. Cette espèce a déjà été mentionnée par LienHARD (1980, p. 374, 376) comme Pseudachorutes spec.

\section{BIBLIOGRAPHIE}

DallaI, R. 1973. Ricerche sui Collemboli. XVI. Stachorutes dematteisi n. gen., n. sp., Micranurida intermedia $\mathrm{n}$. sp. e considerazioni sul genere Micranurida. Redia 54: 23-31.

LienHARD, C. 1980. Zur Kenntnis der Collembolen eines alpinen Caricetum firmae im Schweizerischen Nationalpark. Pedobiologia 20: 369-386.

Massoud, Z. 1967. Monographie des Neanuridae, Collemboles Poduromorphes à pièces buccales modifiées. Biol. Am. Aust. 3: 7-399. 


\section{$2 \mathrm{BHL}$ Biodiversity Heritage Library}

1983. "Deux nouvelles especes du genre Stachorutes Dallai, 1973

(Collembola)." Revue suisse de zoologie 90, 929-934.

https://doi.org/10.5962/bhl.part.82016.

View This Item Online: https://www.biodiversitylibrary.org/item/128827

DOI: https://doi.org/10.5962/bhl.part.82016

Permalink: https://www.biodiversitylibrary.org/partpdf/82016

\section{Holding Institution}

Smithsonian Libraries

\section{Sponsored by}

Biodiversity Heritage Library

\section{Copyright \& Reuse}

Copyright Status: In Copyright. Digitized with the permission of the rights holder.

Rights Holder: Muséum d'histoire naturelle - Ville de Genève License: http://creativecommons.org/licenses/by-nc-sa/3.0/

Rights: https://www.biodiversitylibrary.org/permissions/

This document was created from content at the Biodiversity Heritage Library, the world's largest open access digital library for biodiversity literature and archives. Visit BHL at https://www.biodiversitylibrary.org. 